\title{
Dramatic change in the boundary layer in the symbiotic recurrent nova T Coronae Borealis
}

\author{
G. J. M. Luna ${ }^{1,2,3}$, K. Mukai ${ }^{4,5}$, J. L. Sokoloski ${ }^{6}$, T. Nelson ${ }^{7}$, P. Kuin ${ }^{8}$, A. Segreto ${ }^{9}$, G. Cusumano9 \\ M. Jaque Arancibia ${ }^{10,11}$, and N. E. Nuñez ${ }^{11}$ \\ ${ }^{1}$ CONICET-Universidad de Buenos Aires, Instituto de Astronomía y Física del Espacio (IAFE), Av. Inte. Güiraldes 2620 , \\ C1428ZAA Buenos Aires, Argentina \\ e-mail: gjmluna@iafe.uba.ar \\ 2 Universidad de Buenos Aires, Facultad de Ciencias Exactas y Naturales, Buenos Aires, Argentina \\ 3 Universidad Nacional Arturo Jauretche, Av. Calchaquí 6200, F. Varela, Buenos Aires, Argentina \\ ${ }^{4}$ CRESST and X-ray Astrophysics Laboratory, NASA Goddard Space Flight Center, Greenbelt, MD 20771, USA \\ 5 Department of Physics, University of Maryland, Baltimore County, 1000 Hilltop Circle, Baltimore, MD 21250, USA \\ ${ }^{6}$ Columbia Astrophysics Lab 550 W 120th St., 1027 Pupin Hall, MC 5247 Columbia University, New York, NY 10027, USA \\ 7 Department of Physics and Astronomy, University of Pittsburgh, Pittsburgh, PA 15260, USA \\ 8 University College London, Mullard Space Science Laboratory, Holmbury St. Mary, Dorking RH5 6NT, UK \\ 9 INAF - Istituto di Astrofisica Spaziale e Fisica Cosmica, Via U. La Malfa 153, 90146 Palermo, Italy \\ 10 Departamento de Física y Astronomía, Universidad de La Serena, Av. Cisternas 1200, La Serena, Chile \\ 11 Instituto de Ciencias Astronómicas, de la Tierra y del Espacio (ICATE-CONICET), Av. España Sur 1512, J5402DSP San Juan, \\ Argentina
}

Received 29 June 2018 / Accepted 23 August 2018

\begin{abstract}
A sudden increase in the rate at which material reaches the most internal part of an accretion disk, i.e., the boundary layer, can change its structure dramatically. We have witnessed such a change for the first time in the symbiotic recurrent nova T CrB. Our analysis of XMM-Newton, Swift Burst Alert Telescope (BAT)/X-Ray Telescope (XRT)/UltraViolet Optical Telescope (UVOT), and the American Association of Variable Stars Observers (AAVSO) $V$ - and $B$-band data indicates that during an optical brightening event that started in early $2014(\Delta V \approx 1.5)$ the following occurred: (i) the hard X-ray emission as seen with BAT almost vanished; (ii) the XRT X-ray flux decreased significantly, while the optical flux remained high; (iii) the UV flux increased by at least a factor of 40 over the quiescent value; and (iv) the X-ray spectrum became much softer and a bright, new blackbody-like component appeared. We suggest that the optical brightening event, which could be a similar event to that observed about 8 years before the most recent thermonuclear outburst in 1946 , is due to a disk instability.
\end{abstract}

Key words. binaries: symbiotic - accretion, accretion disks - X-rays: binaries

\section{Introduction}

The interface between a Keplerian accretion disk and the accreting object is known as the boundary layer. This region, in a Keplerian disk, radiates approximately half of the available accretion luminosity, often in X-ray energies, due to its high temperature. The accretion rate determines the optical depth of the boundary layer. A sudden change in the accretion rate can manifest itself through a brightening in optical/UV and a fading in $\mathrm{X}$-rays. Theory predicts a threshold above which the boundary layer will be optically thick to its own radiation and the observed spectrum will be blackbody-like, while below this threshold the spectrum will be that of an optically thin thermal plasma (e.g., Narayan \& Popham 1993; Suleimanov et al. 2014).

$\mathrm{T}$ Coronae Borealis $(\mathrm{T} \mathrm{CrB})$ is one of the four known recurrent novae where the companion is a red giant star, i.e., a symbiotic binary system. In these systems, strong eruptions are triggered by a thermonuclear runaway on the white dwarf (WD) surface after accretion of a critical amount of hydrogen-rich material from the companion. Nova-type outbursts were recorded for $\mathrm{T} \mathrm{CrB}$ in 1866 and 1946 when it reached magnitudes as bright as $V=3$ and became a naked-eye object in the northern sky. It hosts a massive white dwarf, with $M_{\mathrm{WD}}=1.2-1.37 M_{\odot}$ (Belczynski \& Mikolajewska 1998; Stanishev et al. 2004). Unlike most symbiotics, in $\mathrm{T} \mathrm{CrB}$ the M4III (Mürset \& Schmid 1999) donor star fills its Roche-lobe (Belczynski \& Mikolajewska 1998), and accretion thus proceeds through the L1 point into an accretion disk before reaching the WD surface. The orbital period of $227.5687 \pm 0.0099$ days (Fekel et al. 2000) implies an accretion disk that extends out beyond the circularization radius $R_{\text {circ }} \approx 10^{12} \mathrm{~cm}$, i.e., a distance from the WD that has a Keplerian orbit with the same angular momentum that the transferred material had when it passed through the Lagrangian internal point L1 (see Eq. (5) in Wynn 2008).

In terms of its X-ray spectrum, $\mathrm{T} \mathrm{CrB}$ is one of five symbiotic stars with X-ray emission that is sometimes hard and bright enough to be detected with the Neil Gehrels Swift Observatory Burst Alert Telescope (BAT; Gehrels et al. 2004; Kennea et al. 2009). Observations of T CrB with Suzaku and RXTE in 2006 and 2009 showed that the hard X-ray spectrum could be described by a hot, highly absorbed, optically thin thermal 
plasma from the accretion disk boundary layer (Luna et al. 2008; Iłkiewicz et al. 2016). Suzaku, RXTE, and recent NuSTAR observations will be discussed in a forthcoming paper.

The exhaustive historical optical light curve compiled by Schaefer (2014) suggests that T CrB brightened by about 1 mag in $V$ several years $(\sim 8)$ before both recorded recurrent novae outburst. The origin and frequency of these brightening events is unknown, but they perhaps indicate a change in accretion flow onto the WD. Since the onset of the current optical brightening that started in early 2014, referred to as a "super-active" state, Munari et al. (2016) found that the luminosity of the ionizing source has increased, leading to a strengthening of high-ionization emission lines such as He II $\lambda 4686 \AA$ and the nebular radiation, which now overwhelms the red giant continuum. Munari et al. (2016), however, only presented observations through December 2015. The light curve from the AAVSO indicates that the maximum brightness was reached around 4-5 April 2016 (see Fig. 1). Radio observations in May/June 2016 indicate a higher flux than pre-super-active state observations in 2014 (Linford et al. 2016). T CrB was persistently detected with the Swift/BAT since Swift launch in late 2004, until late 2014 when in the span of four 100-day bins, the BAT 14-50 keV flux declined from $\sim 4 \mathrm{mCrab}$ to $\sim 2 \mathrm{mCrab}$, then exhibited a sudden drop to $\sim 0$ (within $1 \sigma$ ) in the following time bin.

In this article we study the super-active state, focusing on the XMM-Newton observation taken about 300 days after the optical maximum. Based on the behavior of the high-energy emission and the nature of the T CrB system, we propose that the superactive state is due to a disk instability. In Sect. 2 we describe our dataset, while in Sect. 3 we present the results from the spectral and timing analysis. In Sect. 4 we present our interpretation.

\section{Observations}

\subsection{Swift}

On January 18, 2017, we started a Swift XRT + UVOT monitoring campaign, first with observations every week, then every two weeks, and during the last year once every month. The Swift/XRT was operated in Photo Counting mode. We extracted source and background count rates from each observation from a circular region with a radius of 20 pixels $\left(\approx 47^{\prime \prime}\right)$ centered on T CrB SIMBAD coordinates $(\alpha=15 \mathrm{~h} 59 \mathrm{~m} \mathrm{30.16 \textrm {s } ;} \delta=$ $\left.+25^{\circ} 55^{\prime} 12.6^{\prime \prime}\right)$. We accounted for the presence of dead columns on the CCDs using the tool xrtlccorr.

\subsection{XMM-Newton}

We observed T CrB on February 23, 2017 (through a DDT time request) using the EPIC camera in Full Window mode, with the medium filter, for $53.8 \mathrm{ks}$ and the OM camera in fast-mode. After removing intervals with high flaring background, the net exposure time was reduced to $38.3 \mathrm{ks}$. Source background spectra and light curves were extracted from circular regions of 32 and 42 arcsec radii, respectively, with the source region centered on $\mathrm{T}$ CrB SIMBAD coordinates and the background in a sourcefree region of the same CCD. We used the RMFGEN and ARFGEN to build the redistribution matrices and ancillary responses. The resulting X-ray spectra were grouped with a minimum of 25 counts per energy bin. For timing analysis, we converted photon arrival times to the solar system barycenter using the SAS task barycen.

We emphasize that grades distribution and offset maps both indicate that the Swift and XMM-Newton observations were not affected by optical loading on the X-ray detector, and the softest $\mathrm{X}$-rays in the spectra are real. Very bright optical sources tend to create spurious X-rays photons, or change the grades and energies of X-ray photons in the case of moderately optically bright sources, and we have verified that this is not the case for $\mathrm{T} \mathrm{CrB}$.

\subsection{Optical/UV photometry}

During each visit with Swift we also obtained UVOT exposures with the UVM2 ( $22246 \AA, F W H M=498 \AA)$ filter. The UVOT light curve was constructed using the uvotproduct tool. Most UVOT observations taken during the current optical brightening are saturated with Vega-magnitudes brighter than about 10 . However, we were able to measure source magnitudes using the readout streak, as detailed by Page et al. (2013). Observations with the optical monitor (OM) on board XMM-Newton with the $V, U, B$, and $W 1$ filters were also saturated.

We also collected multi-epoch photometric observations in the $V$ and $B$ bands from the American Association of Variable Star Observers (AAVSO) to study the X-ray data in the context of the optical state. The observations covered a period of $\sim 5000$ days.

\section{Analysis and results}

The XRT flux reached its lowest value since the launch of Swift during our observing campaign that started in 2017 January. Along with the X-ray fading, the XRT spectra have clearly softened, with a soft component dominating the spectra at energies less than about $1 \mathrm{keV}$. T CrB increased its brightness in UV significantly, from about 15 to brighter than approximately 9.8 UVM2 mag. Figure 1 shows the resulting optical, X-ray, and UV light curves.

On 2017 February 23, well into the super-active state, our deep observation with XMM-Newton showed that the EPIC spectra (Fig. 2) are obviously complex, and can be divided into three energy ranges. Above $3 \mathrm{keV}$, there is a highly absorbed component with a prominent emission line complex in the $6-7 \mathrm{keV}$ range indicative of an optically thin, thermal origin. This is likely the same $\delta$-type component seen in $\mathrm{T} \mathrm{CrB}$ in its normal state (Kennea et al. 2009; Luna et al. 2013). Below $\sim 0.7 \mathrm{keV}$, the spectra are dominated by a soft, unabsorbed component. A blackbody provides a good description of this region. Photons are also detected in the intermediate energy range (0.7$3 \mathrm{keV})$. We fit the spectra in two different ways.

In the first, all components are absorbed by interstellar absorption and local, partial covering absorption that blocks 99.7\% of the emission (model $\mathrm{A}$ in Table 1, see also Fig. 2). The soft X-ray spectra consist of blackbody-like emission (with $T_{\mathrm{bb}}=4 \times 10^{5} \mathrm{~K}$ ) from a region smaller than the surface of the WD, with a spherical surface area of $4.2 \times 10^{7} \mathrm{~km}^{2}$ (in the case of a WD with $1.2 M_{\odot}$ and $R_{\mathrm{WD}}=5 \times 10^{8} \mathrm{~cm}$, this represents approximately $13 \%$ of the surface of the WD). The hard $(0.6 \lesssim$ $E \lesssim 10 \mathrm{keV})$ spectra were consistent with a multi-temperature, cooling flow, with maximum temperature $k T_{\max }=12.9 \mathrm{keV}$. An alternative to this two-component model, where only the $\delta$-component is partially covered by the absorber, yielded a blackbody-component spherical surface area of $1.5 \times 10^{5} \mathrm{~km}^{2}$ (model A').

We also studied an alternative model with three separate thermal components, a soft blackbody-like component, a hard optically thin thermal plasma component, and a medium energy optically thin thermal plasma component to take into account 


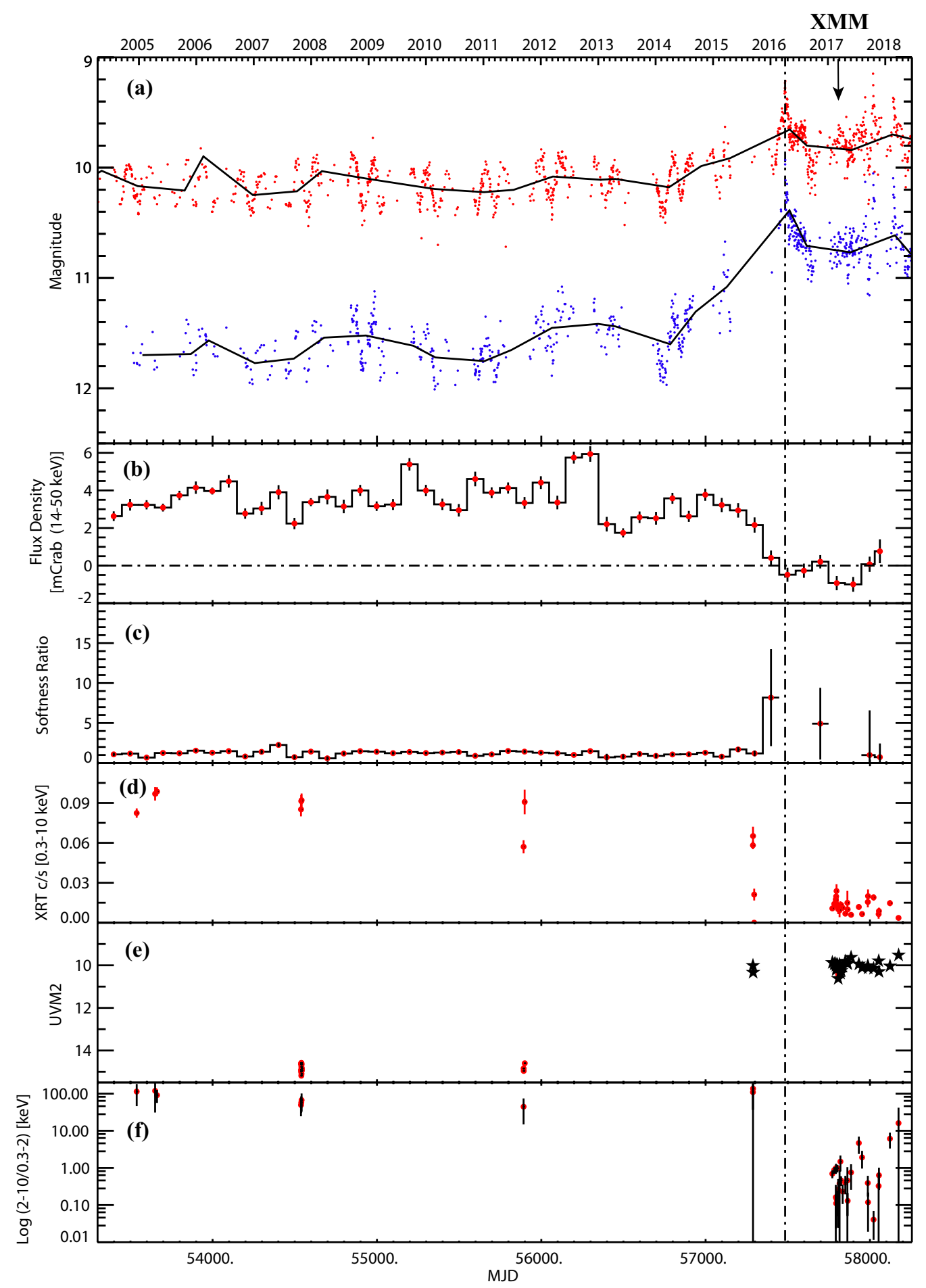

Fig. 1. Panel $a$ : AAVSO $V$-band (red dots) and $B$-band (blue dots) light curves covering the period late 2004-early 2018. The vertical dashed line shows the time of maximum optical brightness. The black solid line shows a curve from a moving average with a mean of 228 days (the orbital period). Panel b: Swift BAT 14-50 keV light curve with 100-day bins. It is evident that the BAT flux started to decay almost simultaneously with the increase in the optical flux, which started around December 2014; it became too faint for its detection around the optical maximum. Panel c: Swift BAT softness ratio (15-25/25-100 keV) with 100-day bins. This ratio steeply increased owing to the softening of the X-ray emission. Panel $d$ : Swift XRT 0.3-10 keV count rate. Panel e: Swift UVOT UVM2-magnitude light curve. Stars indicate magnitudes determined from the CCD readout streak. Even during the rise to optical maximum, the UV flux has already increased dramatically. Panel f: Swift XRT hardness ratio $(2-10 / 0.3-2 \mathrm{keV})$. Since the beginning of the optical brightening, the X-ray emission has become about 100 times softer than before.

photons in the $\sim 0.7-3 \mathrm{keV}$ region. The soft, optically thick components and the medium-energy, optically thin components are modified by mostly interstellar absorption, while the hard, cooling flow is affected by full and partial covering absorbers.
Although in terms of $\chi_{v}^{2}(0.95 / 222$ d.o.f. $)$ this model is also acceptable, parameters such as the absorbing column densities or the covering fraction are unconstrained. The blackbody emitting area in this case was smaller than in model $\mathrm{A}$, with a value of 

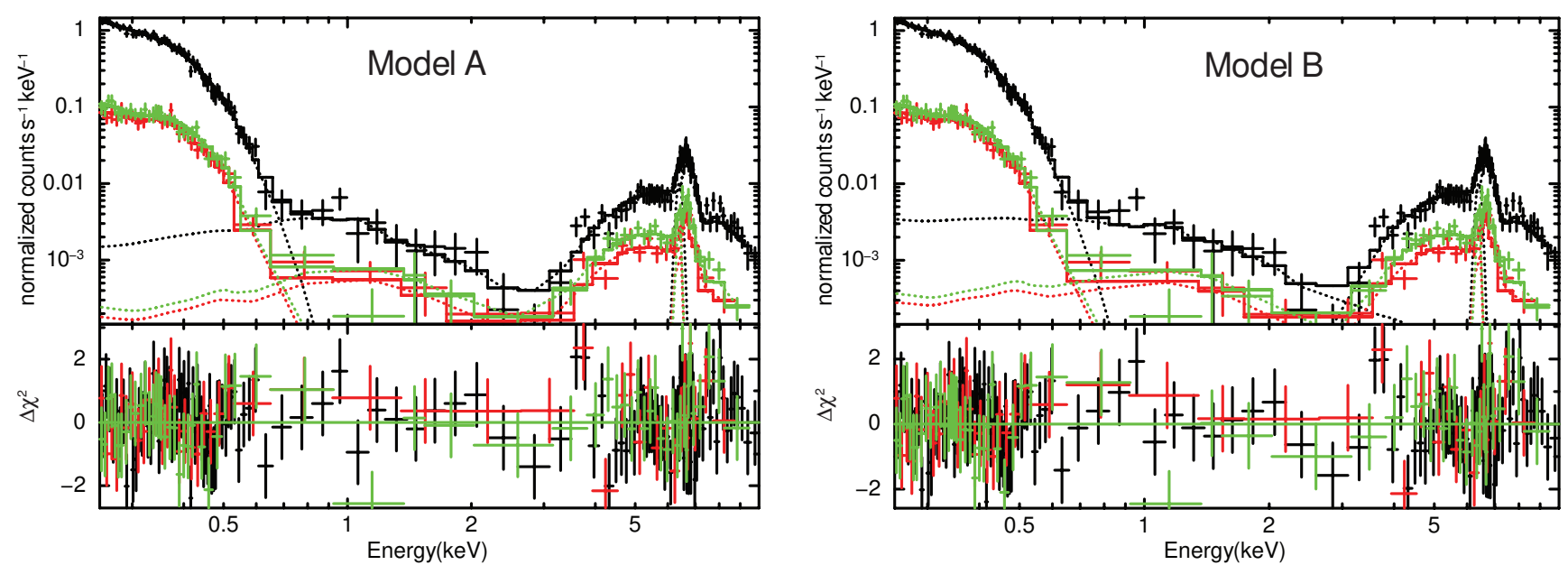

Fig. 2. Left: XMM-Newton pn (black), MOS1 (red), and MOS2 (green) X-ray spectra of T CrB data with the best spectral model (solid line) and the contribution of each model's component (dotted lines). The model consists of a blackbody plus an optically thin cooling-flow and a Gaussian profile centered at the Fe $\mathrm{K} \alpha$ energy of $6.4 \mathrm{keV}$. All components are absorbed by interstellar and local, partial covering absorption (see Table 1 for resulting parameters). Right: alternative model, with a soft blackbody, a hard thermal plasma component, and a medium energy optically thin thermal component to take into account photons in the $\sim 0.6-3 \mathrm{keV}$ region. The soft optically thick and medium energy, optically thin components are modified by mostly interstellar absorption, while the hard, cooling flow is also affected by a partial covering absorber. Both bottom panels show the fit residuals in units of $\chi_{v}^{2}$.

$1.3 \times 10^{5} \mathrm{~km}^{2}$ (covering much less than $0.1 \%$ of the WD surface; model B in Table 1).

The light curves from the XMM-Newton observation, binned at $300 \mathrm{~s}$, show strong variability with fractional amplitudes of 0.32 in the soft $(0.3-0.7 \mathrm{keV}) \mathrm{X}$-ray band and 0.47 in the hard (0.7-10 keV) X-ray band (Fig. 3). The XMM-Newton X-ray light curves did not contain statistically significant periodic modulations. We searched for periodicities in the X-ray light curves (with bins of $300 \mathrm{~s}$ ) in the $0.3-0.7$ and $0.7-10 \mathrm{keV}$ energy ranges by computing the Fourier power spectrum. The log-log power spectrum in the frequency region $f \lesssim 0.0033 \mathrm{~Hz}$ is dominated by red noise. To search for periods with amplitudes in excess of the red noise, we modeled the log-log power spectrum with a simple power law using a least-squares fit (Vaughan 2005). The detection threshold was determined by simply assuming that the model is a good description of the underlying power spectrum, and thus their ratio will be distributed as $\chi^{2}$. We estimated the probability (at $95 \%$ and $99.74 \%$ confidence levels) that at aiven frequency, a large peak would be present in the periodogram by comparing the ratio of modeled to observed power spectrum to the $\chi^{2}$ probability distribution. No peaks in the power spectrum exceeded the detection threshold. None of the power spectra, in any of the selected energy ranges, show periods with a significance greater than $3 \sigma$. Our observations were sensitive to pulsed fraction (following Israel \& Stella 1996) greater than $15 \%$ for $f \lesssim 0.0033 \mathrm{~Hz}$ in the $0.3-0.7 \mathrm{keV}$ range and greater than $22 \%$ for $f \lesssim 0.0033 \mathrm{~Hz}$ in the $0.7-10 \mathrm{keV}$ range.

\section{Discussion}

\subsection{Boundary layer and unstable disk}

From the spectral model fits to the XMM-Newton EPIC spectra, we consider model A to be the most compelling. While the blackbody components in models $\mathrm{A}^{\prime}$ and $\mathrm{B}$ are too small to arise from the WD surface or the accretion disk boundary layer, in model $\mathrm{A}$ it might well correspond to the size of the boundary layer. Changes in strength and character of UV and X-rays, along with optical changes reported by
Table 1. X-ray spectral fitting results.

\begin{tabular}{lcc}
\hline \hline Parameter & Model $\mathrm{A}^{1}$ & Model B \\
\hline$N_{\mathrm{H}}^{a}\left(10^{22} \mathrm{~cm}^{-2}\right)$ & $0.049 \pm 0.002$ & $63_{-34}^{+12}$ \\
$N_{\mathrm{H}}^{b}\left(10^{22} \mathrm{~cm}^{-2}\right)$ & $68 \pm 2$ & $\gtrsim 39$ \\
$\mathrm{CF}^{b}$ & $0.997 \pm 0.001$ & $\gtrsim 0.6$ \\
$k T_{\max }(\mathrm{keV})$ & $12.9 \pm 0.5$ & $13 \pm 5$ \\
$Z / Z_{\odot}$ & $1.5 \pm 0.3$ & $1.5_{-0.6}^{+0.8}$ \\
$\dot{M}\left(10^{-9} M_{\odot} \mathrm{yr}^{-1}\right)$ & $0.16 \pm 0.01$ & $0.17 \pm 0.1$ \\
$N_{\mathrm{H}}^{c}\left(10^{22} \mathrm{~cm}^{-2}\right)$ & $\ldots$ & $\lesssim 0.09$ \\
$k T_{\text {mid }}(\mathrm{keV})^{22}$ & $\ldots$ & $4.5_{-2}^{+25}$ \\
$N_{\mathrm{H}}^{d}\left(10^{22} \mathrm{~cm}^{-2}\right)$ & $\ldots$ & $0.05 \pm 0.01$ \\
$k T_{\mathrm{bb}}(\mathrm{keV})$ & $0.035 \pm 0.001$ & $0.035 \pm 0.002$ \\
$R_{\mathrm{bb}}(\mathrm{km})$ & $1830 \pm 30$ & $103 \pm 10$ \\
$F_{\mathrm{cf}}$ & $61.0 \pm 0.2$ & $65 \pm 2$ \\
$F_{\text {mid }}$ & $\ldots 6000 \pm 1000$ & $0.15 \pm 0.3$ \\
$F_{\mathrm{bb}}$ & 47 & $285 \pm 5$ \\
$L_{\mathrm{cf}}$ & $\ldots$ & 50.5 \\
$L_{\mathrm{mid}}$ & 66800 & 0.12 \\
$L_{\mathrm{bb}}$ & $0.92 / 226$ & $0.95 / 222$ \\
$\chi_{v}^{2} / \mathrm{d}$.o.f. & & 221 \\
\hline
\end{tabular}

Notes. Unabsorbed X-ray flux and luminosity, in units of $10^{-13} \mathrm{ergs} \mathrm{s}^{-1}$ $\mathrm{cm}^{-2}$ and $10^{31} \mathrm{ergs} \mathrm{s}^{-1}$, respectively, are calculated in the $0.3-50 \mathrm{keV}$ energy band for the optically thin thermal components and in the $1 \mathrm{eV}-10 \mathrm{keV}$ band for the optically thick thermal component. Elemental abundances are quoted in units of abundances from Wilms et al. (2000). Luminosity, size of the blackbody emitting region, and $\dot{M}$ are determined assuming a distance of $806 \mathrm{pc}$. ${ }^{(1)}$ TBabs $^{a} \times(\text { partcov } \times \text { TBabs })^{b} \times($ bbodyrad + mkcflow + gaussian). ${ }^{(2)}\left(\right.$ TBabs $^{a} \times(\text { partcov } \times \text { TBabs })^{b} \times($ mkcflow + gaussian $)+$

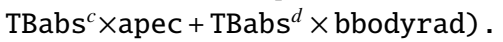

Munari et al. (2016), Zamanov et al. (2016) and Iłkiewicz et al. (2016), demonstrate that after early 2014 the rate of accretion $\dot{M}$ onto the WD increased and the boundary layer became 


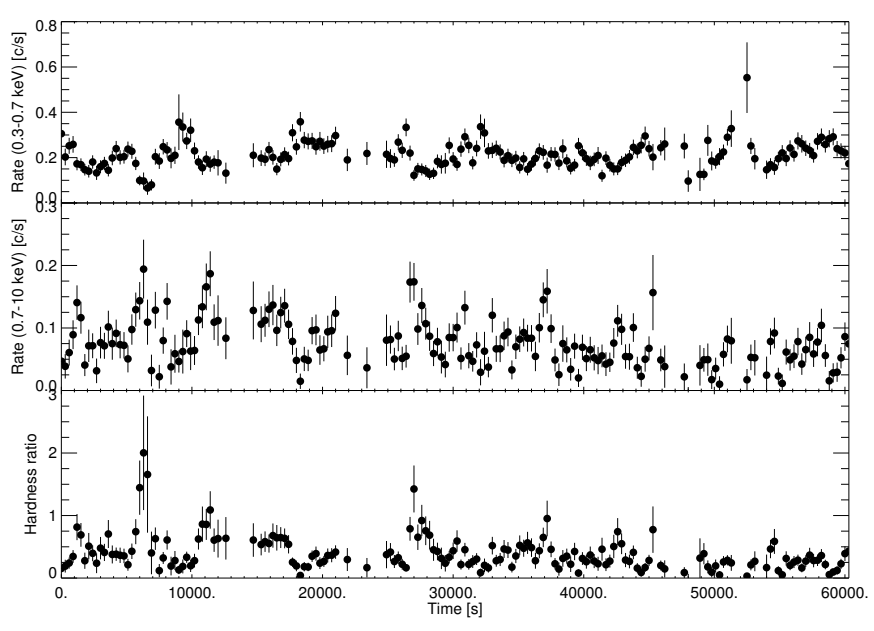

Fig. 3. Background-subtracted XMM-Newton EPIC light curves with bins of $300 \mathrm{~s}$. Top: $0.3-0.7 \mathrm{keV}$. Middle: $0.7-10 \mathrm{keV}$. Bottom: hardness ratio $(0.7-10 \mathrm{keV} / 0.3-0.7 \mathrm{keV})$.

predominantly optically thick. The non-detection of periodicities in the light curves of both the hard and soft X-rays suggests that the emission is not powered by magnetically channeled accretion. The stochastic variability in the hard band is, however, expected if the emission arises in the accretion disk boundary layer. Furthermore, the strong variability observed in the soft band and the small area of the blackbody emitting region derived from spectral model $\mathrm{A}$ indicates that this emission is not powered by thermonuclear burning on the WD surface.

At a distance of $806_{-30}^{+33} \mathrm{pc}$ (as recently determined with Gaia Bailer-Jones et al. 2018), the unabsorbed luminosity of the blackbody-emitting region is $L_{\mathrm{bb}}=A \sigma T^{4}=6.68 \times$ $10^{35}(d / 806 \mathrm{pc})^{2} \operatorname{ergs~s}^{-1}$, with A being the surface area of a sphere with radius $R_{\mathrm{bb}}=1.8 \times 10^{8} \mathrm{~cm}$. The UVM2 fluxes indicate that $L_{\mathrm{UV}}>2 \times 10^{34}(d / 806 \mathrm{pc})^{2} \mathrm{ergs} \mathrm{s}^{-1}$. Assuming that half of the accretion luminosity is radiated in the boundary layer, and that $M_{\mathrm{WD}}=1.2 M_{\odot}$ and $R_{\mathrm{WD}}=5 \times 10^{8} \mathrm{~cm}$, we found that the accretion rate feeding the optically thick portion of the boundary layer was $\dot{M}_{\mathrm{bb}} \approx 6.6 \times 10^{-8}(d / 806 \mathrm{pc})^{2} M_{\odot} \mathrm{yr}^{-1}$. The luminosity of the boundary layer is the sum of the luminosity of the optically thick and optically thin cooling flow components, $L_{\mathrm{BL}}=L_{\mathrm{bb}}+L_{\mathrm{cf}}$. The luminosity of the optically thin portion of the boundary layer, from the cooling flow spectral model in the $0.3-50 \mathrm{keV}$ range, was $L_{\mathrm{cf}} \approx 4.7 \times 10^{32}(d / 806 \mathrm{pc})^{2} \mathrm{ergs} \mathrm{s}^{-1}$. The accretion rate feeding the optically thin portion of the boundary layer was thus $\dot{M}_{\mathrm{cf}}=1.6 \times 10^{-10}(d / 806 \mathrm{pc})^{2} M_{\odot} \mathrm{yr}^{-1}$. These accretion rates are consistent with the expected theoretical values where the boundary layer is optically thick/thin to its own radiation (Patterson \& Raymond 1985; Narayan \& Popham 1993).

The current high optical brightness state appears to be associated with the highest accretion rate on record. The long-term optical light curve of $\mathrm{T} \mathrm{CrB}$ shows a history of different levels of activity (see Stanishev et al. 2004; Munari et al. 2016; Iłkiewicz et al. 2016) and with the exception of the nova eruptions, all of them had lower intensity than the current level. We can rescale, at $d=806 \mathrm{pc}$, the reported accretion rates and compare then with the current level. The $U$-band light curve from May, 1979 to August, 2002 presented by Stanishev et al. (2004) shows that $\mathrm{T}$ CrB was in a low brightness state from JD 2447300-JD 2450000 (August, 1987 through September, 1995), while from JD 2444300 to JD 2447300 (March, 1980 through May, 1988) was in an optical high state. By modeling the International Ultraviolet Explorer (IUE) spectra during the low and high states, Stanishev et al. (2004) found $\dot{M}_{\text {low }}=$ $1.53 \times 10^{-9}(d / 806 \mathrm{pc})^{2} M_{\odot} \mathrm{yr}^{-1}$ and $\dot{M}_{\text {high }}=1.1 \times 10^{-8}$ $(d / 806 \mathrm{pc})^{2} M_{\odot} \mathrm{yr}^{-1}$. Selvelli et al. (1992) analyzed IUE data and suggest that during the decade of the 1980 s the accretion rate was on average $\dot{M}_{\text {low }}=9.6 \times 10^{-9}(d / 806 \mathrm{pc})^{2} M_{\odot} \mathrm{yr}^{-1}$. The optical depth of the boundary layer previous to the Swift launch is unknown. Only short pointed X-ray observation of T CrB ( $\approx 2 \mathrm{ks})$ with Einstein obtained on February 26, 1979 were reported by Cordova et al. (1981), with a $0.1-4.5 \mathrm{keV}$ luminosity of $7.2 \times 10^{30}(d / 806 \mathrm{pc})^{2} \mathrm{erg} \mathrm{s}^{-1}$. In this energy range, T CrB was even fainter than the current X-ray state. It is unknown, however, if a hard X-ray component existed in the past.

The current brightness state, with $\dot{M} \gtrsim 6.6 \times 10^{-8}$ $(d / 806 \mathrm{pc})^{2} M_{\odot} \mathrm{yr}^{-1}$ being the highest on record, dramatically changed the structure of the boundary layer, making it mostly thick to its own radiation. NUSTAR observations in Luna et al. (in prep.) support this interpretation. Theory predicts that the transition from mostly optically thin to mostly optically thick should occur at accretion rates of about $10^{-9} M_{\odot} \mathrm{yr}^{-1}$ for a $1 M_{\odot}$ WD (Popham \& Narayan 1995) or even lower values as proposed by (Suleimanov et al. 2014). The accretion rate before the optical brightening was therefore likely smaller than a few $10^{-9} M_{\odot} \mathrm{yr}^{-1}$.

Phenomenologically, the simultaneous quenching of the hard $\mathrm{X}$-rays, with the remarkable increase in UV flux is exactly what is observed in well-known dwarf novae in outburst. Also, as has been observed in some dwarf novae (Wheatley et al. 2003), in $\mathrm{T}$ CrB the maximum temperature of the cooling flow dropped from $k T_{\max }=57 \pm 10 \mathrm{keV}$, as observed with Suzaku in September 2006 (Luna et al. 2008), to $k T_{\max }=12.9 \pm 0.5 \mathrm{keV}$ during the XMM-Newton observation reported here. The origin of the residual hard X-ray emission during outburst and the decrease in $k T_{\max }$ are still a matter of debate and could be related to an accretion disk corona or the presence of an additional cooling mechanism (see Mukai 2017, for a detailed discussion).

The enhancement of accretion rate in the accretion disk must be due to an increased mass transfer rate from the donor, a disk instability, or both. Although irradiation is cited as a potential cause of enhanced mass transfer in some interacting binaries, it is unlikely to explain the super high state of T CrB. First, the irradiating flux is low: Munari et al. (2016) used the infrared light curves to infer a temperature increase of the heated side of $80 \mathrm{~K}$, or about a $2 \%$ increase compared to the unirradiated side, implying an irradiating flux of $\sim 8 \%$ of the intrinsic stellar flux. Second, Büning \& Ritter (2004) concluded that irradiation of a giant donor cannot result in mass transfer cycles. Finally, the timescale on which the envelope of the giant responds to changing irradiation is many thousands of years, according to the same authors.

In a steady-state disk, the effective temperature as a function of disk radius is given by $T(R)=(3 G / 8 \sigma \pi)^{1 / 4} \dot{M}^{1 / 4} M_{\mathrm{WD}}^{1 / 4} R^{-3 / 4}$ (Eq. (5.43) in Frank et al. 2002, with $R \gg R_{\mathrm{WD}}$ ) and has to be greater than $\sim 10^{4} \mathrm{~K}$, enough to keep $\mathrm{H}$ ionized. In $\mathrm{T} \mathrm{CrB}$, with a WD mass of $1.2 M_{\odot}$ and a mass accretion rate $\dot{M} \sim 6.6 \times$ $10^{-8} M_{\odot} \mathrm{yr}^{-1}$ (determined from the XMM-Newton observation), the steady-state disk would extend out to about $R \approx 0.75 R_{\odot}$. Given the size of circularization radius in $\mathrm{T} \mathrm{CrB}$, it is highly unlikely that the entire disk can remain in a high state (with $T \gtrsim 10^{4} \mathrm{~K}$ ). If the region in the vicinity of the circularization radius is cold and unstable with mostly neutral $\mathrm{H}$ and low viscosity, then the accreted matter would accumulate near that region until a disk instability develops, allowing the material to flow inwards. A similar inside-stable, outside-unstable hybrid accretion disk has been discussed for the recurrent novae RS Oph by Wynn (2008) in an attempt to explain how mass is transferred 
during quiescence periods, given the low accretion rate determined from X-ray observations in quiescence (Nelson et al. 2011). In this scenario, disk instability outbursts in the outer parts of the disk are infrequent, with recurrence time on the order of hundreds of years. Recently, Bollimpalli et al. (2018) studied a similar scenario where the amount of material necessary to trigger a thermonuclear outburst in RS Oph every 20 years is delivered to the WD in a series of disk-instability outbursts, with optical brightening amplitudes of about 1 mag. A similar scenario could be at work in $\mathrm{T} \mathrm{CrB}$.

\subsection{X-ray absorption}

X-ray observations from the past decade indicate that the absorbing column was very high during that time (Luna et al. 2008; Kennea et al. 2009; Iłkiewicz et al. 2016), completely covering the source (Luna et al., in prep.) and absorbing all X-rays with energies lower than $\sim 2-3 \mathrm{keV}$. This might lead us to suspect that the blackbody-like spectral component could have been permanently emitting at the current level, but was only detected when there was a favorable sight. However, optical spectra during the past decade (see Munari et al. 2016) indicate that, with the exception of the current super-active state, the intensity of highly ionized emission lines such as HeII $\lambda 4686$ has been low and the optical continuum weak, in contrast to what is observed now in the super-active state, simultaneous with the appearance of the blackbody-like component. If blackbody-like X-ray emission had been present but absorbed, we would have expected very strong optical line and continuum emission from the absorbing material.

A partial covering absorber is sometimes inferred for $\delta$-type symbiotics. A covering fraction of $99.7 \%$ seems extreme and uncomfortably close to $100 \%$; however, a model with a full covering absorber implies that the soft X-ray emission would be blocked completely. While we cannot exclude a geometrical explanation for this covering fraction, we have also explored a possible alternative. In high accretion rate, non-magnetic $\mathrm{CVs}$, accretion disk wind features are routinely observed in the UV spectra, and perhaps also in the optical spectra (Matthews et al. 2015). It therefore makes sense to ask if the accretion disk wind can be responsible for the partial covering absorption we observe in $\mathrm{T} \mathrm{CrB}$ in high state in particular, and in $\delta$-type symbiotic stars in general. In a line-driven wind, the radiation force is much greater than that due to Thomson scattering because of the combined effect of resonant lines (Castor et al. 1975) with a force multiplier in the range of 1000-4000. This makes a CV with an accretion rate of about $1.0 \times 10^{-8} M_{\odot} \mathrm{yr}^{-1}$ effectively superEddington (Drew \& Proga 2000). Moreover, since T CrB harbors a high-mass white dwarf, its Eddington ratio is higher than that of CVs with a typical white dwarf mass for any given accretion rate. Here we can directly use the inferred luminosity of the optically thick boundary layer of $6.7 \times 10^{35} \mathrm{ergs} \mathrm{s}^{-1}$, which is about $0.4 \% L_{\text {Edd }}$ for a $1.2 M_{\odot}$ white dwarf. With a typical value of force multiplier, $\mathrm{T} \mathrm{CrB}$ is therefore effectively highly superEddington and an accretion disk wind stronger than typically seen in CVs is likely.

Matthews et al. (2015) surveyed the literature of accretion disk winds in CVs and found that wind mass loss rates of $1-$ $10 \%$ were typical for an accretion rate of $10^{-8} M_{\odot} \mathrm{yr}^{-1}$. We have therefore opted to use this as a fiducial accretion rate, and a wind $10^{-9} M_{\odot} \mathrm{yr}^{-1}$ as the fiducial value for the wind mass loss rate. Their model (see a schematic in Fig. 3 of Matthews et al. 2015) has a biconical geometry with an X-shaped cross section, with wind being launched from $4-12 R_{\mathrm{wd}}$. The launch radii may be particularly uncertain as wind is launched from the innermost regions of the disk in other simulations (e.g., Dyda \& Proga 2018b).

We performed the following order-of-magnitude estimate of the resulting absorbing column. We express the mass loss rate in the units of $10^{-9} M_{\odot} \mathrm{yr}^{-1}$ as $\dot{M}_{\mathrm{w},-9}$; for $\dot{M}_{\mathrm{w},-9}=1.0$, the mass loss rate is $2.0 \times 10^{24} \mathrm{~g} \mathrm{yr}^{-1}=6.3 \times 10^{16} \mathrm{~g} \mathrm{~s}^{-1}$. We further express the characteristic radius of the wind where the line of sight to the X-ray emitting region crosses it in units of $10^{9} \mathrm{~cm}$ as $r_{\mathrm{w}, 9}$, and the wind velocity at the same point in units of $1000 \mathrm{~km} \mathrm{~s}^{-1}$ as $v_{\mathrm{w}, 8}$. Dividing the mass loss rate by $4 \pi r v$ for $r_{\mathrm{w}, 9}=1.0$ and $v_{\mathrm{w}, 8}=1.0$, we obtain a mass column density expected from a wind of $5.0 \times 10^{-2} \mathrm{~g} \mathrm{~cm}^{-2}$, or $N_{\mathrm{H}}^{\text {wind }} \sim 5 \times 10^{22} \mathrm{~cm}^{-2}$.

Given our estimate for the accretion rate in $\mathrm{T} \mathrm{CrB}$ in the high state of $6.6 \times 10^{-8} M_{\odot} \mathrm{yr}^{-1}, \dot{M}_{\mathrm{w},-9}=3.0$ would be a plausible assumption. According to Matthews et al. (2015), it takes the wind about $100 R_{\mathrm{wd}}$ to obtain its final velocity, and it has poloidal velocities of less than $100 \mathrm{~km} \mathrm{~s}^{-1}$ within a few $R_{\mathrm{wd}}$, so we can try $v_{\mathrm{w}, 8}=0.1$. Then $r_{\mathrm{w}, 9}=2.2$ would result in the observed $N_{\mathrm{H}}$ value of $6.8 \times 10^{23} \mathrm{~cm}^{-2}$. This admittedly crude estimate suggests that the accretion disk wind is a plausible origin of the local absorber in $\mathrm{T} \mathrm{CrB}$ in high state.

However, our Model A suggests a partial covering absorber with a covering fraction of $99.7 \%$, and the remaining $\sim 0.3 \%$ experiences hardly any absorption at all $\left(4.5 \times 10^{20} \mathrm{~cm}^{-2}\right.$, or less than a thousandth of the column of the partial covering absorber, and this may well be dominated by the interstellar medium). While the 3D simulation of Dyda \& Proga (2018a) does show a clumpy wind, the density contrast between the clumps and interclump regions is only of order 10. In general (i.e., regardless of whether the absorber is the accretion disk wind or something else), we have not identified a natural explanation for a near $100 \%$ covering fraction and a factor of 1000 density contrast.

The scattering model for $\mathrm{CH}$ Cyg of Wheatley \& Kallman (2006) may provide a possible alternative to our best-fit spectral model with its extraordinarily high absorption covering fraction. This model requires low-density matter that gets highly ionized, and a clear line of sight to that region. Such a region could exist in the polar cavity of the wind. For this region to remain highly ionized, it cannot be optically thick to X-rays from the central source, which also limits its scattering efficiency. Combined with geometrical factors, it appears plausible for this region to scatter $\sim 0.3 \%$ of the total luminosity into our line of sight.

In a normal state there are problems for the accretion disk wind as the origin of $\mathrm{X}$-ray absorbers in all $\delta$-type symbiotic stars. One problem is that we would expect a strong inclination angle dependence, with little absorption when a system is seen pole-on. The other is the accretion rate dependence of the wind mass loss rate. The numbers appear to work for $\mathrm{T} \mathrm{CrB}$ in the high state. In a normal state, the accretion rate may only be a few times $10^{-9} M_{\odot} \mathrm{yr}^{-1}$, hence the wind mass loss rate is expected to be on the order of $10^{-10} M_{\odot} \mathrm{yr}^{-1}$ at most, yet $N_{\mathrm{H}}$ in excess of $10^{23} \mathrm{~cm}^{-2}$ is seen in $\mathrm{T} \mathrm{CrB}$ in a normal state. If all X-ray absorbers in $\delta$-type symbiotics are due to accretion disk wind, we would expect a stronger dependence on the accretion rate. Similarly, this model may have difficulty explaining the high $N_{\mathrm{H}}$ values seen in lower X-ray luminosity $\delta$-type systems.

At the time of submission the current optically bright state continues. Our finding that the accretion-disk boundary layer around the $>1.2 M_{\odot}$ WD in $\mathrm{T} \mathrm{CrB}$ transitioned from primarily optically thin to primarily optically thick when the accretion rate rose from $\sim 10^{-9} M_{\odot} \mathrm{yr}^{-1}$ to $\sim 10^{-8} M_{\odot} \mathrm{yr}^{-1}$ (assuming $d=806 \mathrm{pc}$ ) challenges theoretical boundary-layer models, which must also explain BL transitions at much lower accretion rates for some DNe in outburst. 
Acknowledgements. We acknowledge the anonymous referee for the usefu comments that helped to improve the quality of this manuscript. This work is based on observations obtained with XMM-Newton an ESA science mission with instruments and contributions directly funded by ESA Member States and NASA. We thank the entire Swift team for accepting and planning our multiple Target-of-Opportunity requests. We thank Norbert Schartel for approving the XMM-Newton DDT request. We acknowledge with thanks the variable star observations from the AAVSO International Database contributed by observers worldwide and used in this research. We thank Ulisse Munari for the helpful discussions about the current super-active state. GJML and NEN are members of the CIC-CONICET (Argentina) and acknowledge support from grant ANPCYT-PICT 0478/14. GJML also acknowledges support from grant PIP-CONICET/2011 \#D4598. JLS acknowledges support from NASA grants NNX15AF19G and NNX17AC45G.

\section{References}

Bailer-Jones, C. A. L., Rybizki, J., Fouesneau, M., Mantelet, G., \& Andrae, R. 2018, AJ, 158, 58

Belczynski, K., \& Mikolajewska, J. 1998, MNRAS, 296, 77

Bollimpalli, D. A., Hameury, J. M., \& Lasota, J. P. 2018, MNRAS, 481, 5422

Büning, A., \& Ritter, H. 2004, A\&A, 423, 281

Castor, J. I., Abbott, D. C., \& Klein, R. I. 1975, ApJ, 195, 157

Cordova, F. A., Mason, K. O., \& Nelson, J. E. 1981, ApJ, 245, 609

Drew, J. E., \& Proga, D. 2000, New Astron., 44, 2

Dyda, S., \& Proga, D. 2018a, MNRAS, 475, 3786

Dyda, S., \& Proga, D. 2018b, MNRAS, 478, 5006

Fekel, F. C., Joyce, R. R., Hinkle, K. H., \& Skrutskie, M. F. 2000, AJ, 119, 1375

Frank, J., King, A., \& Raine, D. J. 2002, Accretion Power in Astrophysics, 3rd edn., 398

Gehrels, N., Chincarini, G., Giommi, P., et al. 2004, ApJ, 611, 1005
Iłkiewicz, K., Mikołajewska, J., Stoyanov, K., Manousakis, A., \& Miszalski, B. 2016, MNRAS, 462, 2695

Israel, G. L., \& Stella, L. 1996, ApJ, 468, 369

Kennea, J. A., Mukai, K., Sokoloski, J. L., et al. 2009, ApJ, 701, 1992

Linford, J., Weston, J., \& Chomiuk, L. et al. 2016, ATel, 9153

Luna, G. J. M., Sokoloski, J. L., \& Mukai, K. 2008, in RS Ophiuchi (2006) and the Recurrent Nova Phenomenon, eds. A. Evans, M. F. Bode, T. J. O'Brien, \& M. J. Darnley, ASP Conf. Ser., 401, 342

Luna, G. J. M., Sokoloski, J. L., Mukai, K., \& Nelson, T. 2013, A\&A, 559, A6

Matthews, J. H., Knigge, C., Long, K. S., Sim, S. A., \& Higginbottom, N. 2015, MNRAS, 450, 3331

Mukai, K. 2017, PASP, 129, 062001

Munari, U., Dallaporta, S., \& Cherini, G. 2016, New Astron., 47, 7

Mürset, U., \& Schmid, H. M. 1999, A\&AS, 137, 473

Narayan, R., \& Popham, R. 1993, Nature, 362, 820

Nelson, T., Mukai, K., Orio, M., Luna, G. J. M., \& Sokoloski, J. L. 2011, ApJ, 737, 7

Page, M. J., Kuin, N. P. M., Breeveld, A. A., et al. 2013, MNRAS, 436, 1684

Patterson, J., \& Raymond, J. C. 1985, ApJ, 292, 535

Popham, R., \& Narayan, R. 1995, ApJ, 442, 337

Schaefer, B. E. 2014, in Am. Astron. Soc. Meet. Abstr., 223, 209.01

Selvelli, P. L., Cassatella, A., \& Gilmozzi, R. 1992, ApJ, 393, 289

Stanishev, V., Zamanov, R., Tomov, N., \& Marziani, P. 2004, A\&A, 415, 609

Suleimanov, V., Hertfelder, M., Werner, K., \& Kley, W. 2014, A\&A, 571, A55

Vaughan, S. 2005, A\&A, 431, 391

Wheatley, P. J., \& Kallman, T. R. 2006, MNRAS, 372, 1602

Wheatley, P. J., Mauche, C. W., \& Mattei, J. A. 2003, MNRAS, 345, 49

Wilms, J., Allen, A., \& McCray, R. 2000, ApJ, 542, 914

Wynn, G. 2008, in RS Ophiuchi (2006) and the Recurrent Nova Phenomenon, eds. A. Evans, M. F. Bode, T. J. O’Brien, \& M. J. Darnley, ASP Conf. Ser., 401,73

Zamanov, R., Semkov, E., Stoyanov, K., \& Tomov, T. 2016, ATel, 8675 